\title{
Skin Thermal Effect by FE Simulation and Experiment of Laser
}

\section{Ultrasonics}

\author{
Chunhui Li, Sinan Li, Zhihong Huang, Wenbin Xu \\ Division of Mechanical Engineering and Mechatronics, \\ University of Dundee, Dundee DD1 4HN \\ C.Li@Dundee.ac.uk, S.T.Li@Dundee.ac.uk, Z.Y.Huang@Dundee.ac.uk, \\ X.X.Wenbin@Dundee.ac.uk
}

Key Words: Laser Ultrasonics, Finite Element Analysis (FEM), Thermal Effect, Skin.

\begin{abstract}
Laser ultrasonics opened possibilities to measure thermal and mechanical property of skin which occupies an essential position and is beneficial in industrial and medical applications. This paper focuses on the thermal effect in the thermal section of the laser ultrasonic technique. A transient thermal analysis is developed and promoted to simulate the interaction between the laser pulse and human skin, using a multilayered finite element model (FEM). Chicken leg had been used and irradiated by $\mathrm{KrF}$ laser, the thermal reactions were detected and recorded by a thermal camera. By comparison, the thermal result of experiments and simulation matches.
\end{abstract}

\section{Introduction}

The study and research of the mechanical properties of different materials occupies an essential position in many engineering and industrial applications. Laser ultrasonics uses a short laser pulse as a remote ultrasound input to excite thermal expansion, and then induces ultrasonic waves on surfaces, which contains the information of elastic properties. This technology has been widely used in industry to detect the surface condition of metallic materials and obtain relative mechanical properties of coatings [1-5].

Laser ultrasonics has potential to quantify mechanical properties of skin for diagnosis and accurate assessment of skin diseases [6-7]. In skin laser ultrasonics, the study of thermal interaction between a laser pulse and skin becomes a very essential part for the following two reasons:

Firstly, it is of great importance to understand the relationships between increased temperature and laser energy along with other parameters (e.g. shape and duration) in terms of the laser safety. Subsequently, the range of safe laser energy and its relative parameters should be defined in order to avoid skin ablation even skin damage.

Secondly, the amplitude of laser generated surface waves increases with increasing laser energy. It is important that the laser energy should be kept sufficient to produce ultrasonic waves which are readily detectable by available measurement tools such as interferometers and high frequency ultrasound transducers which allows the generated surface waves to be recorded and analysed .

The finite element model provides a clear and detailed process of the thermal effect in skin laser ultrasonics. The previous work shows that the thermal effect of laser-skin interaction is subject to the thermal properties of tested materials and the parameters of laser pulses including the energy, 
shape, pulse duration and focus spot [6]. However, no experiments support the results of finite element model. Thus, it is significant to compare the results of a finite element model and experiments in order to quantify the effectiveness of computer modeling, and to analyze the thermal section of skin laser ultrasonics in real conditions.

This paper mainly focuses on the thermal section of skin characterization by the laser ultrasonic technique. At the beginning, there is a discussion about the thermal results from a finite element simulation in order to provide a theoretical reference and then we introduce the experiment of thermal section of laser ultrasound to list and compare the theoretical and experiment results.

\section{Thermal Analysis}

Skin has three layers: the epidermis, the dermis and subcutaneous fat. Each of them has dramatically different structures, mechanical properties (Table 1) and physiological functions. It is assumed that the geometry of skin can be treated as constant in a small scale and mechanical and thermal properties are considered as constant in this paper.

\begin{tabular}{|l|l|l|l|}
\hline & Epidermis & Dermis & Fat \\
\hline Density $\left(\mathrm{kg} \cdot \mathrm{m}^{-3}\right)$ & 1200 & 1200 & 1000 \\
Specific Heat $\left(\mathrm{J} \cdot \mathrm{kg}^{-1} \cdot \mathrm{K}^{-1}\right)$ & 3.590 & 3.300 & 1.900 \\
Thermal conductivity $\left(\mathrm{W} \cdot \mathrm{m}^{-1} \cdot \mathrm{K}^{-1}\right)$ & 0.24 & 0.45 & 0.19 \\
Young's Modulus $(\mathrm{Pa})$ & 136000 & 80000 & 34000 \\
Poisson's Ratio & 0.499 & 0.499 & 0.499 \\
Thermal Expansion Coefficient $\left(K^{-1}\right)$ & 0.0003 & 0.0003 & 0.00092 \\
\hline
\end{tabular}

Table 1 Mechanical and thermal properties of three layers of skin [7]

In the finite element thermal analysis, PLANE55 is employed as a plane element with a two-dimensional thermal conduction capability. The element has four nodes with a single degree of freedom-temperature, at each node. An axisymmetric boundary condition is applied on the boundary of the model in the direction of the laser beam in order to simplify the solution.

In the meshing part, the element size is set up and increases gradually from epidermis to subcutaneous fat. The thickness of epidermis, dermis and subcutaneous fat are assumed as $0.08 \mathrm{~mm}$, $1.5 \mathrm{~mm}$ and $10 \mathrm{~mm}$, in addition, the element size is $0.005 \mathrm{~mm} \times 0.005 \mathrm{~mm}, 0.02 \mathrm{~mm} \times 0.02 \mathrm{~mm}$ and $1 \mathrm{~mm} \times 1 \mathrm{~mm}$ for epidermis, dermis and subcutaneous fat respectively, and the radius of the model is $20 \mathrm{~mm}$. Figure 1 illustrates the schematic diagram of the laser-irradiated model. 

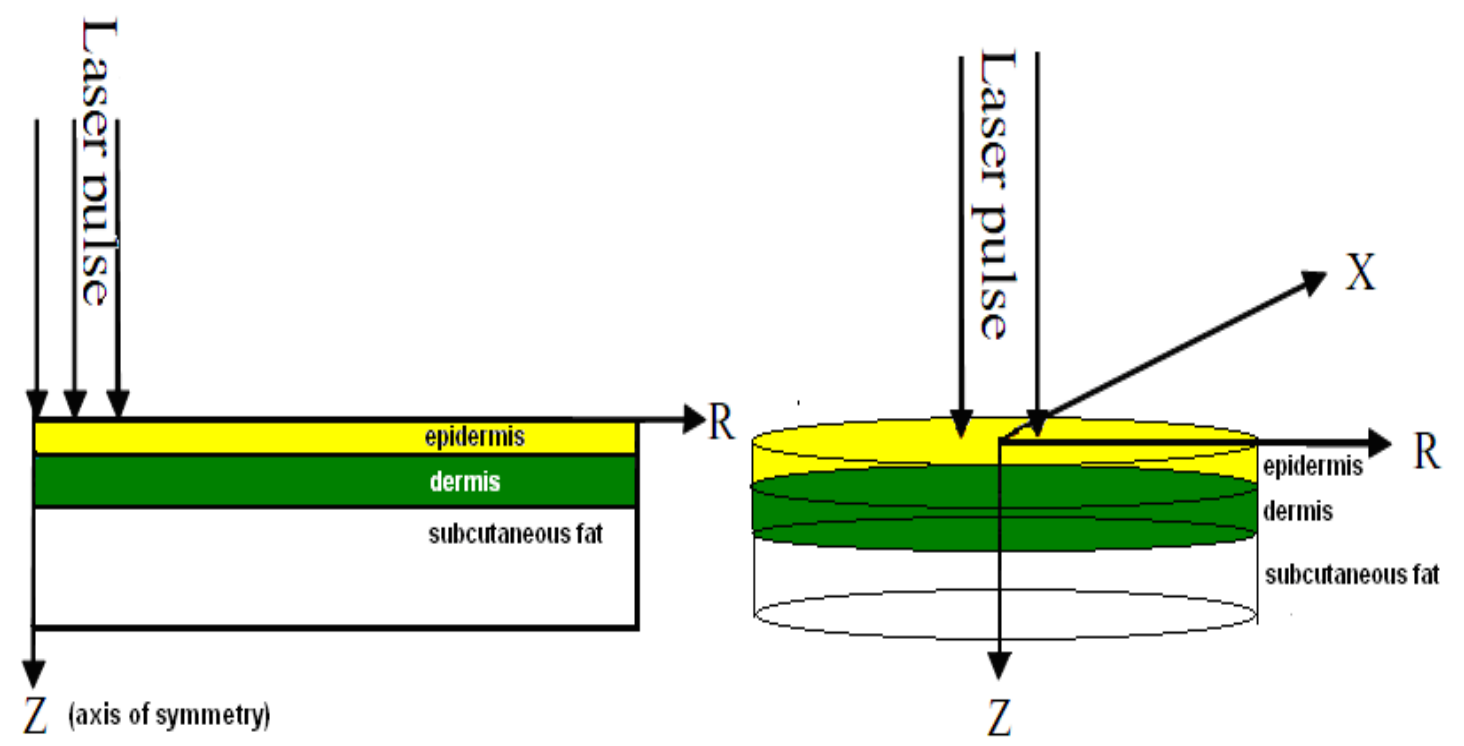

Figure 1 Schematic diagram of laser-irradiated skin model, left shows the model in simulation; right shows the real analyzed model

At the surface of the model, the laser energy is assumed to be a heat generation concentrated on the affected area. Eq.1 expresses the thermal conductive equation in three layers and the boundary condition at the surface of the skin model.

$$
-\left.k_{e} \frac{\partial T_{e}(r, z, t)}{\partial z}\right|_{z=0}=\mu_{\mathrm{a}} * I_{0} * \exp \left(-\frac{r^{2}}{r_{0}^{2}}\right) * \frac{t}{t_{0}} \exp \left(-\frac{t}{t_{0}}\right) * \exp \left(-\mu_{a} \cdot z\right)
$$

Where $K_{e}$ means the conductivity of epidermis; $\mu_{\mathrm{a}}$ is the absorption coefficient of skin $\left(\mathrm{mm}^{-1}\right)$; $I_{0}$ is the irradiance on the surface of tissue $\left(\mathrm{W} \cdot \mathrm{mm}^{-2}\right)$. This equation also contains the spatial and temporal distribution of the Gauss beam as we are using in the experiment, where $r_{0}$ is the beam radius and $\mathrm{t}_{0}$ is the rise time of the Gauss beam, $\mathrm{z}$ is the along the axis of symmetry.

In this simulation the conditions are set as close as possible to the conditions of the experiments with the initial temperature set at $289 \mathrm{~K}$. The laser source in the simulation is assumed to be a $\mathrm{KrF}$ laser with $248 \mathrm{~nm}$ wavelength. The laser energy is $24.7 \mathrm{~mJ}$, and rise time of pulse is $10 \mathrm{~ns}$ and the spot size is $0.5 \mathrm{~mm}$. The time step is set up in the range of $1 \mathrm{~ns}$ for the duration of the laser pulse interaction and it is permitted to increase after the interaction period. The temperature distribution can be plotted in Figure 2. 
TEMPERATURE

$(\mathrm{K})$

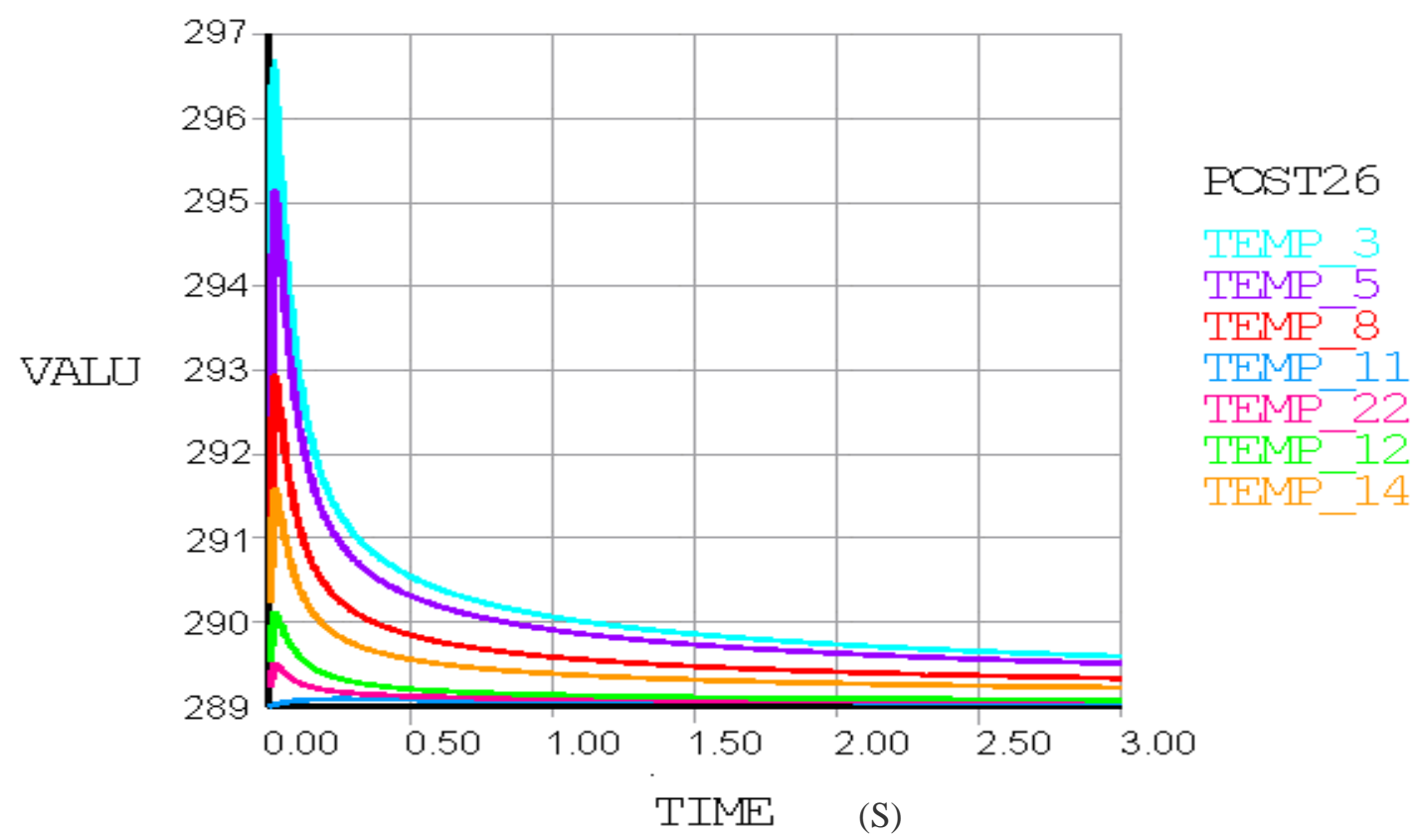

Fig. 2, Temperature in the radial direction at different distance to the center of laser spot of $24.7 \mathrm{~mJ}$

In Figure 2, we can get the temperature distribution along the surface of the skin model from the central laser irradiation to the points far away from the laser source. All points have different level of temperature increase, among which the central point suffers the fastest and highest temperature increase, from $289 \mathrm{~K}$ to $296.8 \mathrm{~K}$. Then, the temperature declines slowly and levels off at a value of 286 Kelvin. It also shows that with the length increasing to the laser source, the peak value of temperature is cut down dramatically, however they all follow the same trend of dropping.

\section{Experiment}

In the experiment of the thermal section of skin laser ultrasonics, a chicken leg is irradiated by a laser pulse to record the temperature field by a Jade infrared thermal camera. The laser device provides the source of the laser beam, and its output energy is acquired by the computer. The optical medium in the laser generator is $\mathrm{KrF}$ gas, and the wavelength of the laser beam is $248 \mathrm{~nm}$ which locates in the ultraviolet section of the electromagnetic spectrum. The laser energy is selected to be $300 \mathrm{~mJ}$ which is maintained as a constant during the experiment and the output energy is verified by attenuation from $20 \%$ to $100 \%$. The energy detector was used to record the energy of the laser pulse that arrived at the tissue surface which is totally different from the theoretical value set up from the computer. In this test the shape of the laser spot is rectangular with a constant energy distribution in the width direction and of Gaussian distribution in the length direction.

The thermal camera used is an infrared camera by Cedip Infrared systems. The camera can image the infrared section of the electromagnetic spectrum with windowing capabilities. The camera is a high performance, highly sensitive camera with a fast frame rate and can record the image in $320 \times 240$ pixels. Using the camera software, Altair, the frequency of imaging can be programmed and the integration time modified to an increment on $1 \mu \mathrm{s}$.

The first step of the experimental procedure consists of setting up the laser parameters in the controlling computer. The energy of the laser beam is controlled using the PC and is varied gradually using the attenuator. The laser pulse irradiated the chicken leg and the temperature was recorded by the thermal camera and the data transferred to the PC for further processing. At the end 
of the test the energy detector is used to record the energy of the laser which varies from the theoretical values due to attenuation from the optical system.

Figure 3 demonstrates a typical result of the temperature distribution in the infrared wavelength and graphs of temperature evolution under irradiation of the specific laser energy, which is directly extracted from the thermal camera.

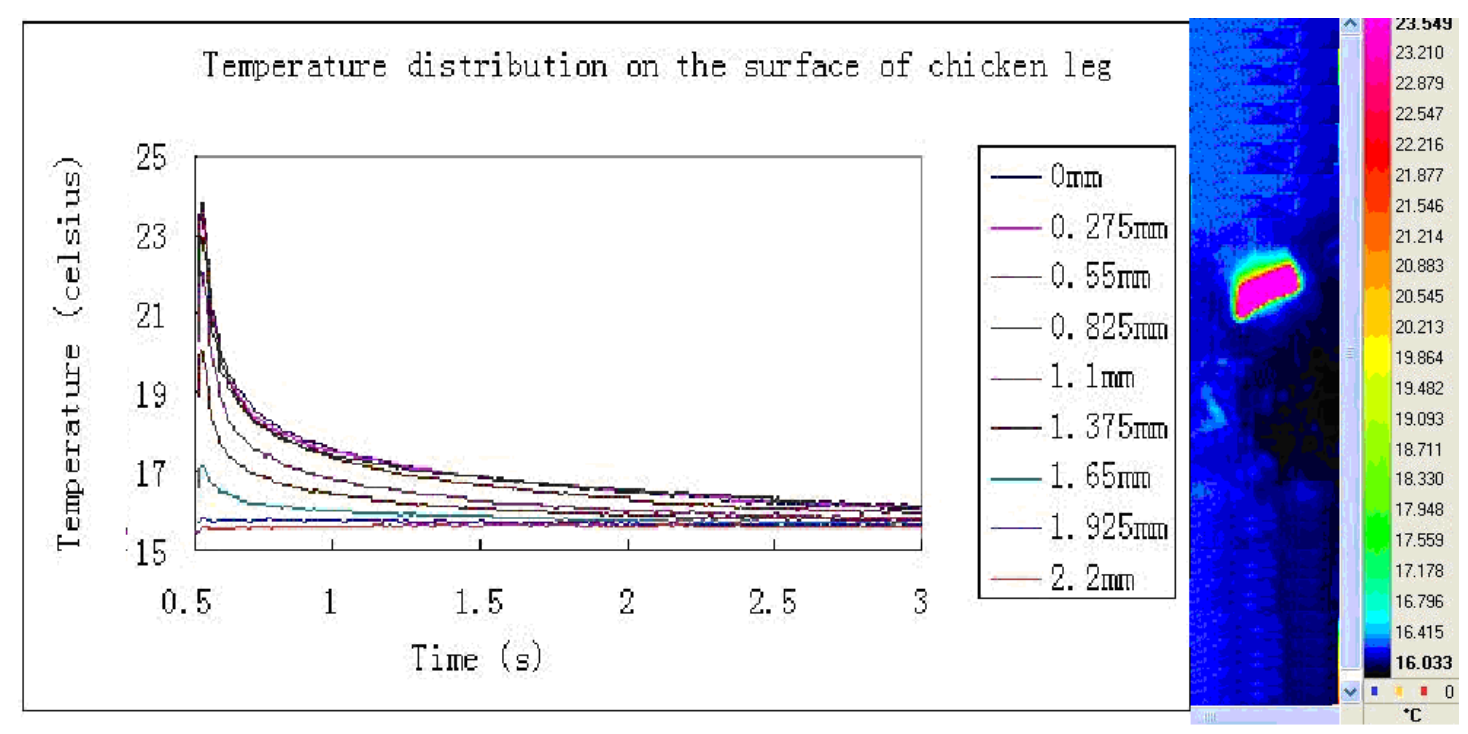

Fig. 3, Diagram of temperature distribution and temperature evolution on chicken leg with laser energy of $24.7 \mathrm{~mJ}$, the highest temperature happens at laser spot center and values $296.3 \mathrm{~K}$.

From Fig. 3, it can be concluded that the temperature evolution of chicken leg shows the exact same pattern that increases sharply due to the temporal distribution of the laser pulse and then drops down slowly as the heat diffuses into the material. The maximum value of temperature is mainly dependent on the laser energy. The highest and fastest temperature increasing point occurs in the center of the laser beam. With the length from the center increased, the temperature will rise slowly due to the diffusion of heat, with a relatively lower peak value.

\section{Conclusions}

It can be concluded that the temperature increment at the center of the laser spot is dependent on the laser pulse and is proportional with laser energy. This point is also becoming the most importance point when discussing the thermal section of skin laser ultrasonics, because it has the maximum temperature increase. It is essential to control this temperature rise to be under the level of laser damage and ablation. From comparison, the result from the simulation has a good match with the experiment data and we can assume that the skin parameters are similar to the ones from chicken skin.

In the future, mechanical section of skin laser ultrasonics will be studied, since it is of importance to relate central temperature rise with amplitude of surface wave together, and analyse the possible safe and functional range of laser energy and parameters. 


\section{Reference}

1. Baiqiang $\mathrm{Xu}$, Xiaowu Ni, Zhonghua Shen Numerical simulation of laser-generated ultrasound by the finite element method. Journal of Applied Physics, Vol. 95 (2004), p2116-p2122.

2. Abbate.A, Russell.W, Goldman.J, Kotidis.P, Berndt.CC Nondestructive determinination of thickness and elastic modulus of plasma spray coatings using laser ultrasonics, Review of Progress in QNDE, Vol. 18 (1999), p373-p380.

3. Bai.T.H, Pei. L.W. and Fang Q.P, Experimental studies of directivity patterns of laser generated ultrasound in neutral glasses, Ultrasonics Vol. 33 (1999), Issue. 6, p429-p436.

4. Davies.S.J, Edwards.C, Taylors.G.S, Palmer.S.B, Laser-generated ultrasound: its properties, mechanisms and multifarious applications, J.Phys.D: Appl.Phys, Vol. 26 (1993), p329-p348

5. Jijun Wang, Baiqiang $\mathrm{Xu}$ and Jian $\mathrm{Lu}$ et al. Influence of transparent coating thickness on thermalelastic force source and laser-generated ultrasound waves. Applied Surface Science (2009). p3-p10

6. Adele LEtang and Zhihong Huang. FE simulation of laser generated surface acoustic wave propagation in skin. Ultrasonics Vol. (2006) 44 p1243-p1247.

7. Jiang.S.C, Ma.N, Li.H.J, Zhang.X.X, Effects of Thermal Properties and Geometrical Dimensions on Skin Burn Injuries, Vol. 28(2002), 713-717.

8. Mansy HA, Sandler RH and Royston TJ. Excitation and propagation of surface waves on a viscoelastic half-space with application to medical diagnosis. Journal of Acoustical Society of America, Vol. 106 (1999), p3678-p3686. 